\title{
Knowledge Management and Emerging Information Technologies: A Review and Prospectus
}

\author{
Ding $\mathrm{Li}^{1, \mathrm{a}}$, Zhiyang $\mathrm{Jia}^{1, \mathrm{~b}^{*}}$ and Yijin Shi ${ }^{1, \mathrm{c}}$ \\ ${ }^{1}$ Tourism and Culture College of Yunnan University, Lijiang, China \\ adingli.ynu@gmail.com, bjdyjazz@gmail.com, ’shiyijin@ynnu.edu.cn
}

Keywords: Knowledge management; Information technology; Web-based; Web 2.0

\begin{abstract}
This paper provides a brief introduction of information technologies concerning knowledge management that applied wildly recently. The presentation of technologies is not studied in detail, but rather a starting point for the researchers to discuss how these emerging technologies such as mobile apps, ubiquitous computing, social networks, technologies related to the Web 2.0, and semantic web could be used in the context of development policy training and knowledge management now and in the future. The paper also tries to categorize individual technologies into technology trends or scenarios of technology development, rather than explain each of the application or technology separately.
\end{abstract}

\section{Introduction}

Knowledge management is currently receiving outstanding attention, from both academics and practitioners, and is being addressed by a broad range of academic literature and popular press [1]. Knowledge management is regarded as a key concern of organizations, particularly those who have already redesigned their business processes and embedded a total quality approach into their practices. Major consulting firms are now gearing up to add knowledge management to their lines of business.

This paper explores the phenomenon of knowledge management from the viewpoint of a management academic with a professional background as an information manager. Knowledge management could be defined by the field of information management, of identifying the differences amongst data, information and knowledge, and the implications of these differences for professional education. Knowledge sharing, exchanging and dissemination are elements in a broader theme which is knowledge management. The central purpose of knowledge management is to transform information and intellectual assets into enduring value [2].

\section{Web-based Techniques}

With recent development in Internet technology and its wider acceptance and popularity there are many products available today to facilitate knowledge sharing and coloration using web technologies. The main advantage of web-based technology is it can be deployed in Internet or in intranet within the organization. Internet is also used to create a VPN using internet as medium of data transmission [3]. In this paper, we will discuss more about web based knowledge management technologies here.

Web technologies offer a number of features useful for knowledge management and distribution. These include a rich set of development tools, universally available access, and an easy-to-use interface. Of special attraction is how links between pages can help organize large amounts of information into a hypertext and how Web standards permit the display of various media in an attractive and interactive interface [4].

Web 2.0. According to the WIKIPEDIA [5], the phrase Web 2.0 [6] can refer to a perceived second generation of web-based communities and hosted services - such as social-networking sites, wikis, and folksonomies - which aim to facilitate creativity, collaboration, and sharing between users. 
"Web 2.0 is a knowledge-oriented environment where human interactions generate content that is published, managed and used through network applications in a service-oriented architecture."

Technologies such as weblogs (blogs) social book-marking, wikis, podcasts, RSS feeds (and other forms of many-to-many publishing), social software, and web application programming interfaces (APIs) [7] provide enhancements over read-only websites.

Web 2.0 websites typically include some of the features/techniques presented in the triangle [8] of Fig. 1.

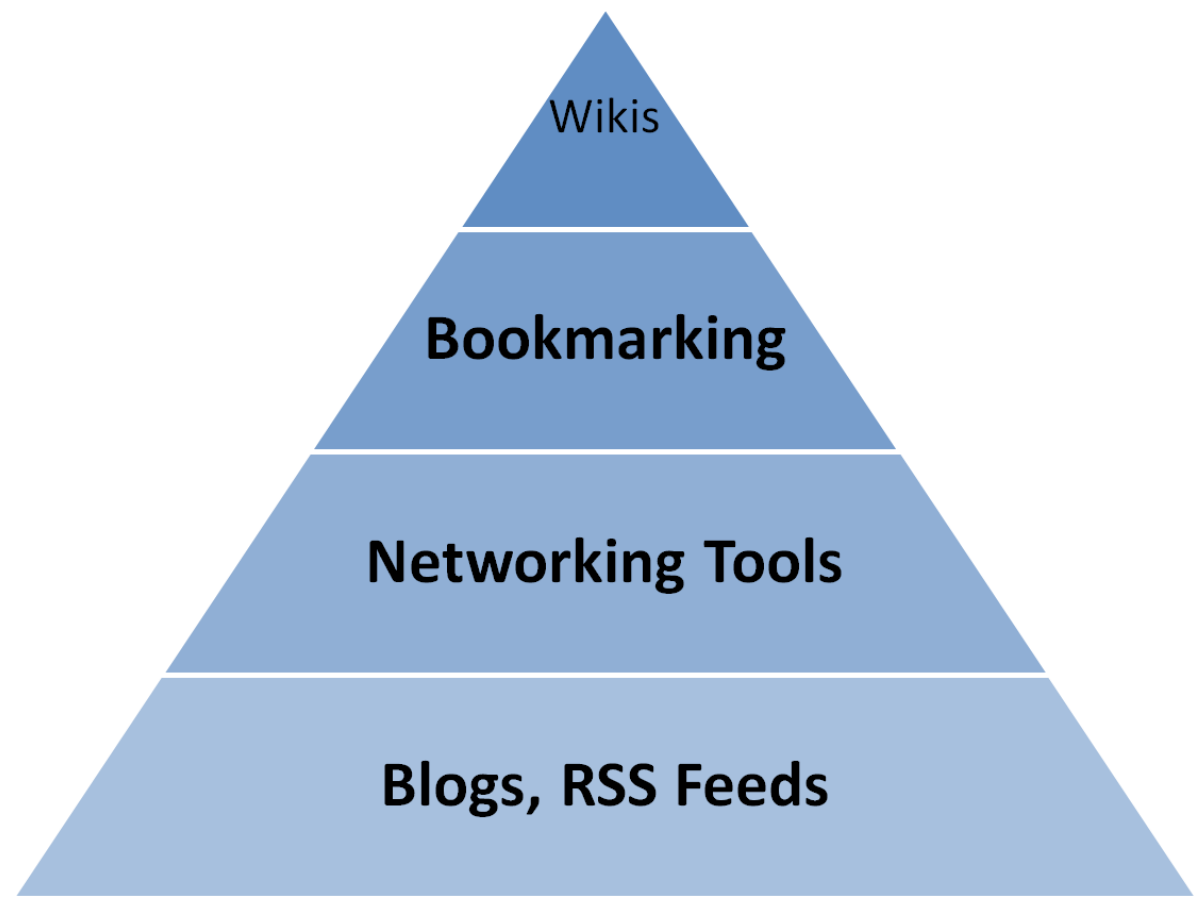

Figure 1. Web 2.0 features triangle

At the base of the Web 2.0 triangle are the blogs - many companies/agencies having their principal officers run internal/external blogs. As an external tool that plugs into a corporate website, it is an excellent tool for thought leadership positioning. Another tool, that's fairly simply to integrate into an existing web presence are RSS feeds.

Moving up the ladder we come to networking tools. Networking can be tapped in two ways. The first and possibly the most rewarding is the creation of web communities. A moderated community that can actually share members' experiences and data is a self-sustaining web site. Plus it's a great platform to promote new ideas and products, for organizing user training and even for getting advance feedback for under-construction features. Another interesting way of using networking is to create alumni online group of an institution/agency.

The next levels of services are built around bookmarking. In a social bookmarking system [9], users save links to web pages that they want to remember and/or share. Social bookmarking sites are popular and useful and they can be bent to a professional context.

With bookmarking, collaboration kicks in and wikis are the next logical step. While it requires an application to house and manage the wiki, once this is set up, organizations will find that wikis are self-sustaining without the overhead of separate teams that intranets normally entail. The wiki is used for knowledge management, project information shaing, news and updates - users have rights for specific sections that they periodically update and each group maintains its own area. The wiki has since cannibalized some of the functions of the intranet and the knowledge management systems since it is always better updated.

Social Networks. The application of relations that link individuals and collectives, have implications for the speed and effectiveness with which knowledge is created and disseminated in organizations Both social networks and knowledge management (KM) are complex, multifaceted phenomena that are as yet imperfectly understood. Not unsurprisingly, our understanding of the 
interface between the two is similarly imperfect and evolving. There are, however, a number of foundational concepts upon which existing thought converges as well as a body of emerging research that offers practical and conceptual guidance for developing the kind of network best suited for managing different kinds of knowledge.

It may be time to put down employees' tools in trying to make the old model of knowledge management work. Social media is a completely new beast that changes many of the rules. In the old world order, knowledge was usually created and stored as a point in time. In the future, organizational policy or insight may not be formed by an individual creating a document that goes through an approval process and is ultimately published. It will likely begin with an online conversation and it will be forever evolving as more people contribute and circumstances change.

Social networks take knowledge and make it highly iterative. It creates content as a social object. That is, content is no longer a point in time, but something that is part of a social interaction, such as a discussion. It easily disassembles the pillars of structure as it evolves. As examples: content in a micro-blogging service can shift meaning as a discussion unfolds; conversations in enterprise social networks that link people and customer data can defy categorization; and internal blogs and their comments don't lend themselves to obvious taxonomy.

\section{Semantic Web}

The Semantic Web [10] will bring structure to the meaningful content of Web pages, creating an environment where software agents roaming from page to page can readily carry out sophisticated tasks for users. The semantic web provides a common standard (RDF) [11] for websites to publish the relevant information in a more readily machine-process able and integrity form.

Semantic Web promises to make Web-accessible data more amenable to machine processing. The Semantic Web is about labeling information so that computer systems can process it more meaningfully. The semantics underlying such annotations usually come from ontologies, which encapsulate agreement among information creators and users with help from common nomenclature and the use of rich knowledge representation.

Just as the Semantic Web is beginning to empower and energize content on the Web, the underlying principles and technologies can energize and enhance the long standing knowledge management discipline. Several frameworks within knowledge management theory set contexts for scientific debate. Some emphasize the knowledge life cycle, others the knowledge product and many researchers have recently begun to emphasize the knowledge and social networking perspective, as previously described. The rapid adoption of Web 2.0 and Web 3.0 technologies adds to this picture a tight connection between knowledge management, social networks, and various implicit, formal, or powerful semantics.

The latest Semantic Web developments and insights in knowledge management challenge the new era of semantic-based knowledge management systems. Semantic Web tools and applications contribute significantly to knowledge management's performance, providing a definition for flexible reference mechanisms to knowledge objects and knowledge contributors; integration of knowledge creation and use; integral human involvement in information and knowledge management activities; and a definition for and the exploitation of social networks, including social activities and context.

\section{Ubiquitous Computing}

Ubiquitous computing [12] is a post-desktop model of human-computer interaction in which information processing has been thoroughly integrated into everyday objects and activities. As opposed to the desktop paradigm, in which a single user consciously engages a single device for a specialized purpose, someone "using" ubiquitous computing engages many computational devices and systems simultaneously, in the course of ordinary activities, and may not necessarily even be aware that they are doing so. 
Ubiquitous computing can help kick off the recent boom in mobile computing, although it is not the same thing as mobile or wireless computing. Ubiquitous computing encompasses a wide range of technologies, including distributed computing, mobile computing, sensor networks, human-computer interaction, and artificial intelligence. However, it this limited document, these technologies are discussed here together and the concept of mobile computing is emphasized.

In practice, the ubiquitous computing can provide technologies where the environment (an intelligent house, ma-chines like refrigerator, etc.) are adapting to our needs and our portable devises are communicating the systems that are integrated to our environment through technologies like Bluetooth, GPRS, Wi-Fi, GPS, etc. From our needs point of view, a mobile computing devise that is linked to the knowledge management system (semantic web able web community site) and is also linked to our environment (location) through a satellite GPS system, is the most interesting.

For example, the ability to determine the receiver's absolute location allows GPS receivers to perform as a surveying tool or as an aid to navigation to locations that needs to be visited. It also allows to search, gather, produce and share information (pictures, data, comments, etc.), that are location related. It also enhances social networking by pin-pointing the other colleagues of the community (if allowed by the member of the community). This can enhance the community of practice to work together and share meaningful and relevant information related to working sites.

\section{Smart Phones and Apps}

In recent years, smart phones have evolved from unsophisticated communication devices to powerful hand-held processors [13]. Currently, they support mastery and recall a wide range of information that encases our work and social interactions. Latest knowledge management applications can be delivered as native apps running on the device for iPhone, iPad, Android, Windows Phone, Windows Mobile, Windows XP/Vista/7 Classic-style, Windows 8 Metro-style, or running on the server as HTML5 [14] delivered in a browser or part of a hybrid Phone Gap application.

Enterprises have turned to explicit and even conceptualizing on tacit knowledge management to elaborate a systematic approach to develop and sustain the intellectual capital needed to succeed, the knowledge normally attributed to knowledge workers. This is complemented by structural capital, i.e. the structures, technologies, practices put in place by an organization as an attempt to manage their specialist knowledge. Mobile learning would equally come under such an umbrella, enticing knowledge workers and managers within organizations to conduct work in a mobile manner. Android and iOS apps installed in Smart phones can be an invaluable tool that helps people collect process and share all the knowledge.

Knowledge management apps [15], which is view as a business unit sought out a tool to solve a specific, tactical knowledge management problem, in this case, opening up communications (as opposed to establishing a formal, overarching knowledge management program). Both users and IT liked the solution to the tactical problem. Scenarios are a long way from the old model for knowledge management, where users often were unilaterally presented with a complex knowledge management system by IT. One of the driving forces behind Web 2.0 is the virtual office-teams of far-flung experts collaborating online to create a whole greater than the sum of its contributors. Finding the most recent version of a document required extra work as well-resulting in productivity losses and missed deadlines when team members mistakenly worked from the wrong version of a document. Sometimes corporate knowledge remains locked up in employee files and inboxes even when other knowledge management tools are in use. So knowledge management apps can easily solve this problem. And features of knowledge management apps include: Troubleshoot \& resolve IT issues in a few clicks from any smartphone or tablet; Supports all key IT infrastructure technologies - AD, VMware, Backup Exec, Exchange \& more; Delivers real-time alerts and statuses on users' Android, iOS, and Blackberry devices; Remote control with SSH, Telnet, VNC, RDP, proxy via Mobile Admin Server or direct connect. 


\section{Acknowledgement}

The research work is supported by Foundation of Bureau of Science and Technology Lijiang, Yunnan Provincial under Grant No. 1js2013-09 and scientific research foundation of Tourism and Culture College of Yunnan University under Grant No. 2013xy08.

\section{References}

[1] Nada K. Kakabadse, Andrew Kakabadse, and Alexander Kouzmin, Reviewing the knowledge management litera-ture: towards a taxonomy, Journal of Knowledge Management. Vol. 7 Iss: 4, pp.75 - 91, 2003.

[2] James Bishop, Dino Bouchlaghem, Jacqueline Glass, Isao Matsumoto, Ensuring the effectiveness of a knowledge management initiative, Journal of Knowledge Manage-ment, Vol. 12 Iss: 4, pp.16-29, 2008.

[3] C.D. Tarantilis, C.T. Kiranoudis and N.D. Theodorakopou-los, A Web-based ERP system for business services and supply chain management: Application to real-world process scheduling, European Journal of Operational Re-search, Vol. 187 Iss:3, pp. 1310-1326, June 2008.

[4] Alavi, Leidner, Review: Knowledge management and knowledge management systems: Conceptual foundations and research issues, Knowledge Management MIS Quarterly, Vol. 25 No. 1, pp. 107-136, March 2001.

[5] Reid Priedhorsky, Jilin Chen, Shyong K. Lam, et al, Creating, destroying, and restoring value in wikipedia," Proceed-ings of the 2007 international ACM conference on Supporting group work, ACM New York, NY, USA, 2007.

[6] Stephen J. Andriole, "Business impact of Web 2.0 technologies, Communications of the ACM. Vol. 53 Iss 12, pp. 67-79, December 2010.

[7] S Murugesan, Understanding Web 2.0, It Professional. Vol. 9 Iss. 4, pp. 34-41, July-Aug. 2007.

[8] Jonathan Grudin, Enterprise Knowledge Management and Emerging Technologies, Proceedings of the 39th Hawaii International Conference on System Sciences, 2006.

[9] Tony Hammond, Timo Hannay, Ben Lund, and Joanna Scott, Social Bookmarking Tools (I) A General Review, D-Lib Magazine, Vol. 11 No. 4, April 2005.

[10] Anupriya Ankolekar, Markus Krötzsch, Thanh Tran, and Denny Vrandecic, The two cultures: mashing up web 2.0 and the semantic web, Proceedings of the 16th international conference on World Wide Web, ACM New York, NY, USA, 2007.

[11] A Maedche, Ontology learning for the semantic web, Intelligent Systems. Vol. 16 Iss. 2, pp. 72-79, Mar-Apr 2001.

[12] J Hightower, G Borriello, Location systems for ubiquitous computing, Computer. Vol. 34 Iss. 8, pp. 57-66, Aug 2001.

[13] H Falaki, R Mahajan, S Kandula, D Lymberopoulos, R Govindan, Diversity in Smartphone Usage, International Conference on Mobile Systems, 2010:179-194.

[14] J Behr, P Eschler, Y Jung, M Zöllner, X3DOM: a DOM-based HTML5/X3D integration model, Proceedings of Web3d ACM, 2009:127-135.

[15] KE Samuel, ML Goury, A Gunasekaran, A Spalanzani, Knowledge management in supply chain: An empirical study from France, Vol. 20, Iss. 3, pp. 283-306, 2011. 